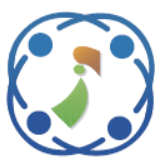

\title{
A New Weighted-learning Approach for Exploiting Data Sparsity in Tag-based Item Recommendation Systems
}

\author{
Noor Ifada ${ }^{1 *} \quad$ Richi Nayak $^{2}$ \\ ${ }^{1}$ Informatics Department, University of Trunojoyo Madura, Indonesia \\ ${ }^{2}$ School of Computer Science, Queensland University of Technology, Australia \\ * Corresponding author's Email: noor.ifada@trunojoyo.ac.id
}

\begin{abstract}
The tag-based recommendation systems that are built based on tensor models commonly suffer from the data sparsity problem. In recent years, various weighted-learning approaches have been proposed to tackle such a problem. The approaches can be categorized by how a weighting scheme is used for exploiting the data sparsity - like employing it to construct a weighted tensor used for weighing the tensor model during the learning process. In this paper, we propose a new weighted-learning approach for exploiting data sparsity in tag-based item recommendation system. We introduce a technique to represent the users' tag preferences for leveraging the weighted-learning approach. The key idea of the proposed technique comes from the fact that users use different choices of tags to annotate the same item while the same tag may be used to annotate various items in tag-based systems. This points out that users' tag usage likeliness is different and therefore their tag preferences are also different. We then present three novel weighting schemes that are varied in manners by how the ordinal weighting values are used for labelling the users' tag preferences. As a result, three weighted tensors are generated based on each scheme. To implement the proposed schemes for generating item recommendations, we develop a novel weighted-learning method called as WRank (Weighted Rank). Our experiments show that considering the users' tag preferences in the tensor-based weightinglearning approach can solve the data sparsity problem as well as improve the quality of recommendation.
\end{abstract}

Keywords: Data sparsity, Tag-based item recommendation, Tensor model, User tag preference, Weighted-learning approach, Weighting scheme.

\section{Introduction}

Tag-based systems permit their users to freely annotate their favourite items using tags. Users may annotate an item using various tags as well as repeatedly using a tag for annotating various items. When a user annotates an item using a tag, a $\langle$ user, item, tag $\rangle$ ternary relation is naturally formed. Over time, these ternary relations become the tagging data that can be used to learn the users' past behaviours. The learning results can then be used to generate ranked list of recommended items that the users might like $[1,2]$ or to retrieve items that they are searching for $[3,4]$.

The tag-based recommendation methods based on the tensor models have shown to outperform those of the matrix models due to their capacity of representing the latent relations inherent in tagging data more efficiently [5]. However, these methods commonly suffer from data sparsity problem and compromise accuracy. This happens since users of tag-based systems typically use and annotate only a few tags and items, respectively. Existing methods mostly use the binary interpretation scheme to populating the tensor model, i.e., the observed tagging entries are denoted as " 1 " while the unobserved ones are denoted as "0" [5]. Thus, a tensor model populated with such Boolean scheme usually results in an over-domination of unobserved entries.

Researchers have been proposing to deal with the data sparsity problem occurs in tensor models by implementing a weighted-learning approach that used a weighting scheme to employ both observed and unobserved data in either the modelling or 
learning process. In this ongoing research topic, a weighting scheme naturally regards the observed data as positive feedback. Meanwhile, the non-observed ones are discriminated as negative and/or other types of feedback. In other words, not all of the nonobserved data should be simply regarded as negative feedback.

In this paper, we propose a new weightedlearning approach for exploiting data sparsity in tagbased item recommendation system. We introduce a technique to represent the users' tag preferences that are used for leveraging the weighted-learning approach such that both observed and unobserved entries are taking into account in the learning process. The key idea of the proposed technique comes from the fact that users use different choices of tags to annotate the same item while the same tag may be used to annotate various items in tag-based systems. This points out that users' tag usage likeliness is different and therefore their tag preferences are also different. We then present three novel weighting schemes that are varied in manners by how the ordinal weighting values are used for labelling the users' tag preferences. As a result, three weighted tensors are generated based on each weighting scheme. To implement the proposed weighting schemes for generating item recommendations, we develop a novel weighted-learning method called as WRank (Weighted Rank).

The contribution of this paper is proposing a new weighted-learning approach for tag-based item recommendation. In the approach, we present a technique to generate the users' tag preferences that are used to formulate three distinct weighting schemes, resulting in three weighted tensors. We then develop a new tag-based item recommendation method to employ the proposed weighting schemes. Our experiments show that considering the users' tag preferences in the tensor-based weighting-learning approach can solve the data sparsity problem as well as improve the quality of recommendation.

The rest of this paper is organized as follows. Section 2 describes the previous works related to the tag-based recommendation and weighted-learning approach. Section 3 details our proposed weightedlearning approach, while Section 4 describes the experiment setup. Section 5 shows the empirical results as well as the discussions based on the experiments. The conclusion of this paper is presented in Section 6.

\section{Related work}

\subsection{Tag-based recommendation using tensor model}

A tag-based recommendation is an application that incorporates tags in the recommendation process to improve the recommendation quality [1]. In this case, users of such application are given the opportunity to freely label items of their interest using tags and forming tagging data. One efficient solution to represent the $\langle$ user, item, tag ternary relationships within the tagging data is by using a tensor model [5]. The model reveals the latent relationships within the multi-dimensions by generating latent factors through tensor factorization process. The latent factors are then used for generating a reconstructed tensor that generated a ranked list of recommendations. Tensor factorization can be categorized as Tucker or Candecomp/Parafac (CP) based techniques. Tucker factorization decomposes a tensor into mode- $n$ product of a core tensor and three factor matrices of each dimension. While CP factorization decomposes a tensor into a sum of component rank-one tensors.

Symeonidis, et al. [6] is among the first researchers that studied the implementation of the tensor model for a tag-based recommendation. They proposed a framework for the tag-based recommendation that implemented HOSVD, i.e., a common factorization of Tucker-based. In our previous work, we developed TRPR [7] to outcompete HOSVD in tag-based item recommendation. Our method implemented various factorization techniques to generate the candidates of recommendations. Afterwards, we implemented a probabilistic technique to re-rank the candidates and the results are used as the final list of recommendations for the target users. Experiment results on Delicious and Last.fm datasets showed that $T R P R$ performs best when it is implemented using CP factorization technique.

Klašnja-Milićević, et al. [8] proposed a tag-based recommendation method that uses ranking with tensor factorization technique for a programming tutoring system. The method tackled the scalability problem by employing a clustering technique to the learners' data. Experiment results showed that the implementation of ranking factorization technique can outperform FolkRank and HOSVD.

Yang, et al. [9] developed the Tagrec-CMTF method for tag recommendation. The method combined the implementation of $\mathrm{CP}$ factorization, tag graph, and also the tag usage of items and users. The results of experiments on Last.fm and Bibsonomy 
datasets showed that Tagrec-CMTF outcompetes $C P$ and PITF methods. However, The nature of the combinatory approach makes the process of profiling and learning stages more complex than the regular factorization based methods.

Tang, et al. [10] proposed a Tucker based approach for tag-based item recommendation. They tried to incorporate the users and items profiles, constructed by implementing a partial-Tucker factorization, within the learning process. Despite its promising results, the approach assumed that the information used to create the profiles always contains a homogonous type of data, which make it difficult to be implemented for the heterogonous problems.

In this paper, we propose a tag-based item recommendation method that implements a $\mathrm{CP}$ factorization technique. We name our method as WRank (Weighted Ranking) as it enhances the capability of the factorization technique for generating a ranked list of recommendation by the implementation of weighted-learning approach (discussed in the next sub-section). We benchmark our proposed method with HOSVD [6] and TRPR [7].

\subsection{Tensor-based weighted-learning approach}

The tensor-based weighted-learning approach can be categorized by how a weighting scheme is used for exploiting the data sparsity. A weighting scheme can be used for: (a) populating the entries of the tensor model used as the learning model, (b) weighing the regularization during the learning process, and (c) weighing the tensor model during the learning process.

Implementing a weighting scheme for building the tensor model is to make sure that both the observed and unobserved data are represented in the learning model. Commonly, a tensor model is built by simply interpreting the data based on a binary scheme, i.e., the observed data is regarded as positive entries and weighted as " 1 " while letting the rest as " 0 ". As a result, the tensor becomes very sparse since the number of observed entries is usually a lot smaller than those of the unobserved ones. Rendle and Schmidt-Thieme [11] proposed the PITF method for tag recommendation. The method used the set-based scheme, pairwise factorization, and AUC-based optimization. The scheme discriminates the weight of the observed and non-observed tagging data in the tensor model. In this case, the initial tensor model is built as pairwise sets of the positive entries of the observed data and the negative entries of the nonobserved ones, in which the former always have higher weight than the latter. Despite the promising results compared to $\mathrm{CP}$ and Tucker based methods, PITF oversimplifies its weighting interpretation towards the non-observed data. Moreover, the implementation of the AUC-based pairwise learning does not differentiate the mistakes occur at the top and bottom list of recommendations. In our previous work, we developed Do-Rank [12] to outcompete PITF in tag-based item recommendation. The method used the User Tag Set (UTS) scheme, CP factorization, and DCG-based optimization. The scheme determines the positive entries of observed data in the same way as the set-based, however, it further discriminates the non-observed data such that not all of them are regarded as the negative entries. Anyhow, both aforementioned methods proved that a portion of unobserved entries is effecting the recommendation quality in the weighted-learning based methods. Lim and Kim [13] developed the RateRT method for tag-based item recommendation. The method employed the tag-weighting scheme, HOSVD factorization, and MSE-based optimization. The scheme populates the tensor learning model based on a linear combination of the rating-based and emotion-based tag weights. Lim and Kim [14] then tried to extend their aforementioned work by adopting the BM25 technique to construct the tagbased user profile and tag-based item profiles. The recommendation results are ranked using the similarity of the profiles. Meanwhile, Zhang, et al. [15] proposed the TRS method that is based on the improvement of Artificial Fish Swarm Algorithm (AFSA) for tag recommendation. The method used rating-tag scheme, Tucker factorization, and MSEbased optimization. The scheme populates the tensor model according to the weight of the tag in each useritem-tag combination multiplies by the rating given by the user to the item. The three aforementioned methods indeed enrich the tensor learning model, however their implementations always require the availability of both the tagging and rating data.

The regularization is the parameter, commonly set as constant, that can be used to avoid overfitting in the tensor-based optimization process. Hosono, et al. [16] proposed the WTNN method for image restoration that implemented the generalization of Local Color Nuclear Norm (LCNN) regularization function and HOSVD factorization. The method conducts colour image denoising towards the image tensor model. $\mathrm{Mu}$, et al. [17] developed the WTNN2 method for image recovery and video completion that used a weighted tensor nuclear norm regularization function and HOSVD factorization. The regularization is defined as the sum of the weighted nuclear norm of all the tensor frontal slices. 
The weighted-learning approach can also be implemented by distinctly weighing the tensor learning model using a weighted tensor during the learning process. In other words, such an approach always contains two tensors of the same size. Acar, et al. [18] is among the first researchers that studied the implementation of the weighted-learning approach that employs the usage of a weighted tensor. They proposed the $C P$-WOPT method for completing the missing values in the EEG and network traffic data. $C P-W O P T$ is based on $\mathrm{CP}$ factorization and uses the Boolean weighting scheme for building the weighted tensor. The weighting scheme generates each entry of the weighted tensor such that it is a direct bijective mapping of the tensor learning model. The main idea of $C P-W O P T$ is to focus on emphasizing the observed entries and disregarding the unobserved ones. Experiment results showed that $C P-W O P T$ outperformed the INDAFAC method. Filipović and Jukić [19] proposed the Tucker based weighted optimization method for image reconstruction that also used the Boolean weighting scheme for building the weighted tensor. The experiment results were promising, however, the method has not been proven that it can be implemented on a large dataset. Yuan, et al. [20] worked on the TT-WOPT method for image data completion that is based on a tensor-train factorization. The weighted tensor is built using the Boolean weighting scheme and is implemented to the sequences of the three-order image tensor-train models. The main idea of TT-WOPT is to find the factor core tensors of the tensor-train factorization. Iwata, et al. [21] tried to advance the TT-WOPT method for predicting the unknown parts of drug and the new drug therapeutic indications. However, due to the nature of the tensor-train factorization, $T T$ $W O P T$ is naturally very sensitive to the setting of the TT-ranks since each number of ranks must be distinguishingly defined. Moreover, the initial values of the core tensors can influence the performance of $T T-W O P T$.

In this paper, we propose a new weightedlearning approach in which a proposed weighting scheme is used to construct a weighted tensor for differentiating the weight of each entry of the tensor model during the learning process. Our weighting scheme is formulated such that it takes into account the users' tag preferences. We benchmark our method with PITF [11], Do-Rank [12], and CP-WOPT [18]. Note that we adapt PITF for the task of tag-based item recommendation since it was originally built for tag recommendation.

\section{The proposed approach}

The framework of the proposed weightedlearning approach for exploiting data sparsity in tagbased item recommendation system consists of four main stages (Fig. 1): user profiling for building the tensor model, weighting scheme for generating the weighted tensor, weighted-based learning for generating the latent factors, and recommendation generation.

\subsection{User profiling}

User profiling is the process of formally representing the user's past tagging activity that will be used as the learning model. In this paper, we denote $U=\left\{u_{1}, u_{2}, u_{3}, \ldots, u_{Q}\right\}$ as the set of $Q$ users, $I=\left\{i_{1}, i_{2}, i_{3}, \ldots, i_{R}\right\}$ as the set of $R$ items, and $T=$ $\left\{t_{1}, t_{2}, t_{3}, \ldots, t_{S}\right\}$ as the set of $S$ tags. Given the tagging data $A: U \times I \times T$, a vector of $a:(u, i, t) \in A$ denotes that user $u$ uses tag $t$ for annotating item $i$. The ternary relations of tagging data can be modelled as a tensor model $\mathcal{Y} \in \mathbb{R}^{Q \times R \times S}$ where $y_{\text {uit }}$ denotes the valuation of a tagging entry. In this case, the slices of the tensor can be seen as user matrices that store the annotation of items using tags.

The observed tagging data, $A_{o b}$ where $A_{o b} \subseteq A$ and $\left|A_{o b}\right| \ll|A|$, defines that users have annotated items by using tags in the past. The Boolean interpretation scheme [6] is commonly used to build the user profile such that the tagging data is directly interpreted as binary data. In this case, each entry of $y_{\text {uit }} \in[0,1]$ corresponds to the type of the tagging data entries, i.e. if the entry is observed then $y_{\text {uit }}=1$ and otherwise $y_{u i t}=0$.

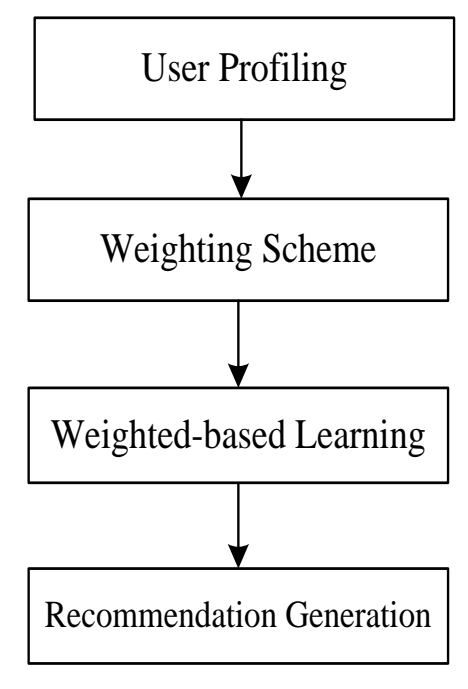

Figure. 1 The framework of the proposed weightedlearning approach for tag-based item recommendation system 


\subsection{Weighting scheme}

Weighting scheme, the main component in a weighted-learning approach, controls the significance of the observed and unobserved entries in the learning process. Given the learning model represented as tensor $\mathcal{Y}$, we store the weighting values in a weighted tensor $\mathcal{W}$. Note that $w_{\text {uit }}$ is a bijective mapping of $y_{\text {uit }}$ that is not changing over the learning process.

\subsubsection{Existing weighting scheme}

The existing weighting scheme, i.e., Boolean scheme, emphasizes the observed entries of $\mathcal{Y}$ using the "1" values, while the unobserved entries are regarded as " 0 " values. The scheme determines the weighting value of $w_{\text {uit }}$ as a direct bijective mapping of the value of $y_{\text {uit }}[18]$ :

$$
w_{\text {uit }}= \begin{cases}1 & \text { if } y_{\text {uit }}=1 \\ 0 & \text { otherwise }\end{cases}
$$

The fact that users can use various tags to annotate an item as well as use a tag to annotate numerous items points out that each user's tag usage likeliness is unique and therefore their tag preferences are also different. We employ this assumption to leverage the weighted-learning approach such that both observed and unobserved entries of $\mathcal{Y}$ are distinctively taking into account in the learning process.

\subsubsection{Proposed user tag preference}

We propose to form the user tag preference by capturing the characteristics of a user's tag usage likeliness through the latent features of the users and tags. In this case, we implement a non-negative matrix factorization (NMF) technique [22] to the mode- $1 Y_{(1)} \in \mathbb{R}^{Q \times R S}$ and mode- $3 Y_{(3)} \in \mathbb{R}^{S \times Q R}$ matricizations of tensor $\mathcal{Y} \in \mathbb{R}^{Q \times R \times S}$ :

$$
\begin{gathered}
Y_{(1)}=A C^{\prime} \\
Y_{(3)}=B D^{\prime}
\end{gathered}
$$

where $A \in \mathbb{R}^{Q \times F}$ and $B \in \mathbb{R}^{S \times F}$ are respectively the latent features of the users and tags of size $F$. Meanwhile, $C \in \mathbb{R}^{R S \times F}$ and $D \in \mathbb{R}^{Q R \times F}$ are the coefficient matrices. The user $u$ tag usage likeliness towards tag $t$ is then calculated as:

$$
l_{u t}=\sum_{k=1}^{F} a_{u k} b_{k t}
$$

where $l_{u t}, a_{u k}$, and $b_{k t}$ are respectively the elements of matrices of the User Tag Usage Likeliness $L \in$ $\mathbb{R}^{Q \times S}$, the User Latent Feature $A \in \mathbb{R}^{Q \times F}$, and the Tag Latent Feature $B \in \mathbb{R}^{R \times F}$. Fig. 2 shows the algorithm of generating the User Tag Usage Likeliness.

Two sets of user tag preferences are formed based on the likeliness scores: (i) User Positive Tag Preference set $L_{u}^{+}$, i.e., the list of tags that the user is possibly interested to use for annotating items, yet it is not explicitly exposed; and (ii) User Negative Tag Preference set $L_{u}^{-}$, i.e., the list of tags that the user does not want to use. The size of the sets is determined by $v$. In this case, $L_{u}^{+}$is created based on the top scores of $L_{u^{*}}$, where $L_{u}^{+} \subseteq T$ such that $\left|L_{u}^{+}\right| \leq$ $v$. While $L_{u}^{-}$is created based on the bottom scores of $L_{u^{*}}$, where $L_{u}^{-} \subseteq T$ such that $\left|L_{u}^{-}\right| \leq v$.

Example: As shown in Fig. 3, the mode-1 and mode- 3 matricizations of tensor $\mathcal{Y} \in \mathbb{R}^{3 \times 4 \times 5}$ result in $Y_{(1)} \in \mathbb{R}^{3 \times 20}$ and $Y_{(3)} \in \mathbb{R}^{5 \times 12}$. The implementation of NMF to $Y_{(1)}$ and $Y_{(3)}$, where $F=$ 2 , results in $A \in \mathbb{R}^{3 \times 2}$ and $B \in \mathbb{R}^{5 \times 2}$. Hence, by calculating $L \in \mathbb{R}^{3 \times 5}$ using Eq. (4) and by choosing $v=2$, the User Positive Tag Preference and User Negative Tag Preference sets are formed as $L_{u_{1}}^{+}=\left\{t_{1}, t_{3}\right\}, L_{u_{2}}^{+}=\left\{t_{2}, t_{4}\right\}, L_{u_{3}}^{+}=\left\{t_{2}, t_{4}\right\}, L_{u_{1}}^{-}=$ $\left\{t_{2}, t_{5}\right\}, L_{u_{2}}^{-}=\left\{t_{1}, t_{3}\right\}$, and $L_{u_{3}}^{-}=\left\{t_{1}, t_{3}\right\}$.

\subsubsection{Proposed weighting scheme}

Based on the results of the proposed User Tag Preference stage, we propose three novel weighting schemes: (1) Quaternary-Tag-Preference, (2) Ternary-Positive-Tag-Preference, and (3) TernaryNegative-Tag-Preference. The bottom side of Fig. 3 shows the examples of the constructed $\mathcal{W}$ based on

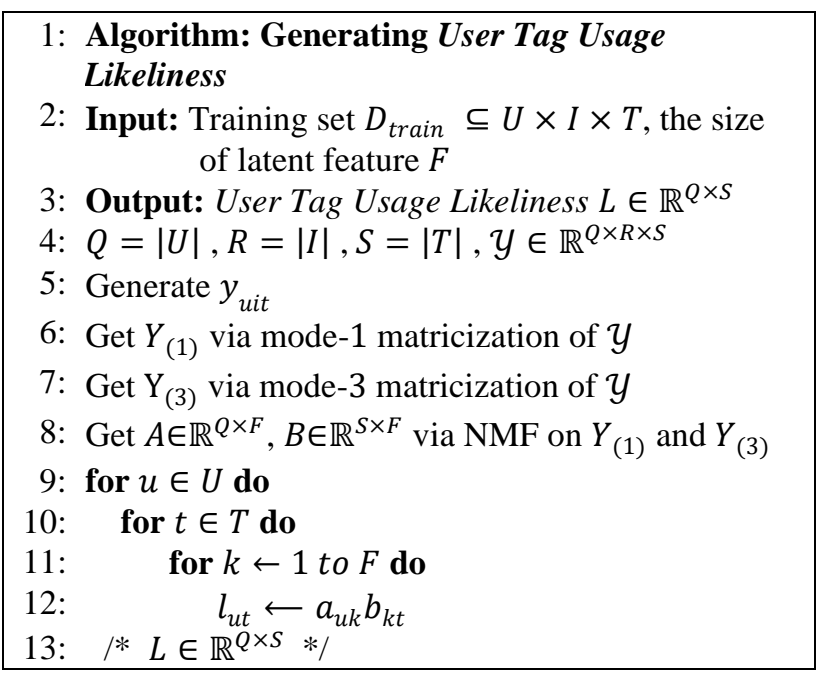

Figure. 2 The algorithm of generating user tag usage likeliness 


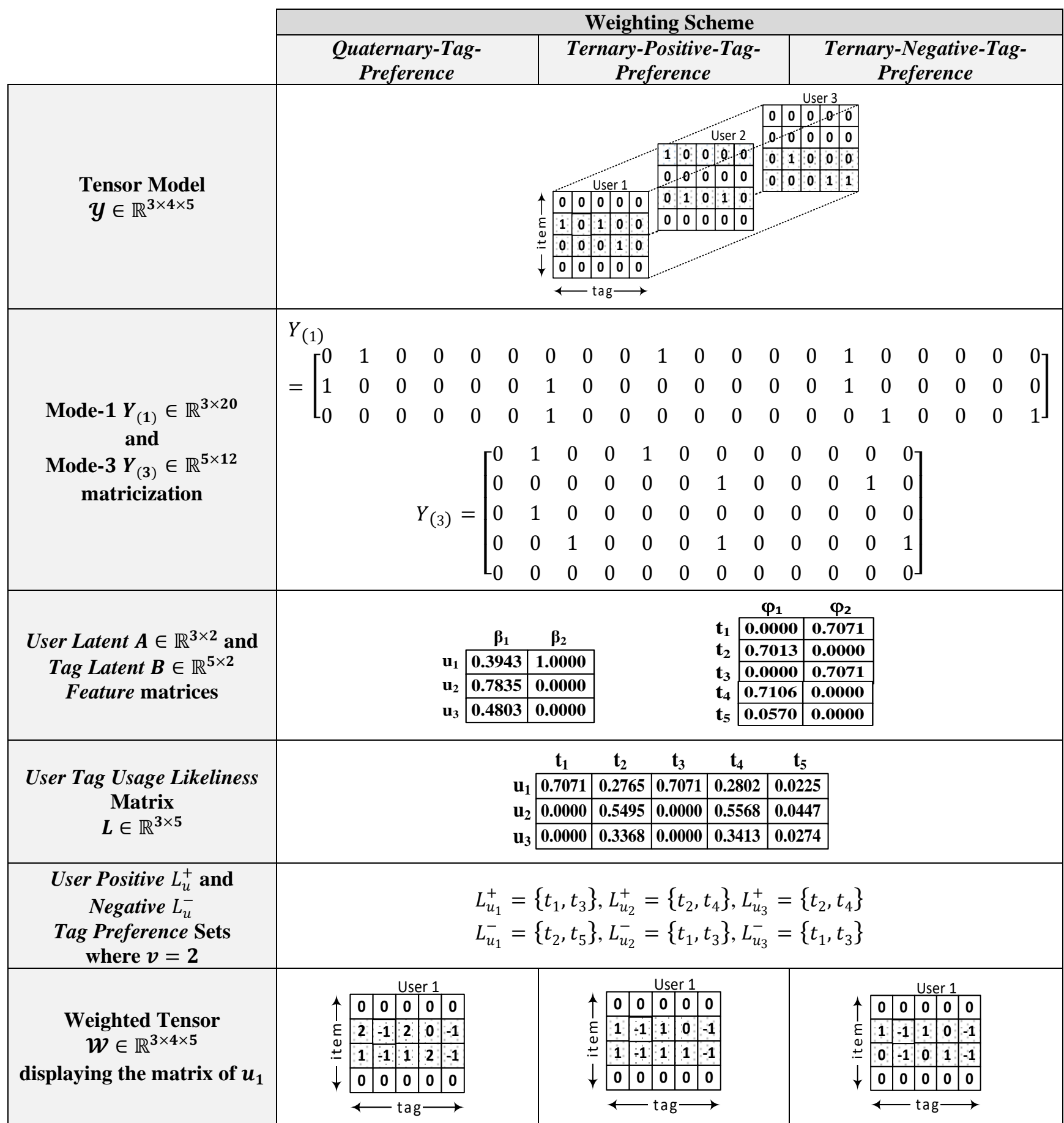

Figure. 3 Examples showing the process of forming the user tag preference and constructing the weighted $\mathcal{W}$ of $u_{1}$

each scheme. Note that, for ease of illustration, the examples only show the $\mathcal{W}$ entries for $u_{1}$.

\section{Quaternary-Tag-Preference weighting scheme:} This scheme generates four ordinal weighting values of $\{2,1,0,-1\}$. The " 2 " value represents the observed entries, whereas " 1 " and " -1 " values represent the unobserved entries that respectively belong to $L_{u}^{+}$and $L_{u}^{-}$. The rest of the entries are denoted as " 0 ". The rule for generating the values of $\mathcal{W}$ entries is formulated as:

$$
w_{\text {uit }}=\left\{\begin{array}{cc}
2 & \text { if } y_{u i t}=1 \\
1 & \text { if } y_{u i t} \neq 1 \wedge t \in L_{u}^{+} \\
-1 & \text { if } y_{u i t} \neq 1 \wedge t \in L_{u}^{-} \\
0 & \text { otherwise }
\end{array}\right.
$$

Ternary-Positive-Tag-Preference weighting scheme: This scheme generates three ordinal weighting values of $\{1,0,-1\}$. The " 1 " value represents the observed entries or unobserved entries that belong to $L_{u}^{+}$, whereas " -1 " value represents the unobserved entries that belong to $L_{\bar{u}}^{-}$. The rest of the 
entries are denoted as " 0 ". This scheme gives equal reward to both the observed entry of $\mathcal{Y}$ and the entry of unobserved $L_{u}^{+}$. It assumes that the user has the same interest to use the tags to annotate items though the latter is not explicitly revealed. The rule for generating the values of $\mathcal{W}$ entries is formulated as follows:

$$
\begin{gathered}
w_{\text {uit }}= \\
\left\{\begin{array}{c}
1 \\
-1
\end{array} \quad \begin{array}{c}
\text { if } y_{\text {uit }}=1 \quad \vee\left(y_{u i t} \neq 1 \wedge t \in L_{u}^{+}\right) \\
0
\end{array} \quad\right. \text { otherwise }
\end{gathered}
$$

Ternary-Negative-Tag-Preference weighting scheme: This scheme generates three ordinal weighting values of $\{1,0,-1\}$. The " 1 " value represents the observed entries, whereas " -1 " value represents the unobserved entries that belong to $L_{u}^{-}$. The rest of the entries are denoted as " 0 ". This scheme disregards the entries of unobserved $L_{u}^{+}$. It assumes that though the user has a positive preference for the list of tags, yet the fact that it was not revealed simply means that the user does not want to generally use them to annotate all items. In other words, the observed entries of $y$ themselves sufficiently represent the user's positive tag preference. The rule for generating the values of $\mathcal{W}$ entries is:

$$
w_{\text {uit }}=\left\{\begin{array}{cc}
1 & \text { if } y_{\text {uit }}=1 \\
-1 & \text { if } y_{\text {uit }} \neq 1 \wedge t \in L_{u}^{-} \\
0 & \text { otherwise }
\end{array}\right.
$$

\subsection{Weighted-based learning}

Weighted-based learning is the stage where the learning process deals with the sparsity problem by focusing only on the known entries in the tensor model [18]. Here, we named our proposed weightedlearning method as WRank (Weighted Ranking).

\subsubsection{Objective function and factorization technique}

The learning model of a weighted-learning approach is optimized as a weighted loss function. The objective function is formulated as:

$$
L(\Theta)=\sum_{u=1}^{Q} \sum_{i=1}^{R} \sum_{t=1}^{S} w_{u i t} \cdot \ell\left(y_{u i t}, \hat{y}_{u i t}\right)(8)
$$

where $\ell\left(y_{\text {uit }}, \hat{y}_{\text {uit }}\right)$ is a loss function while $y_{\text {uit }}$ is the valuation of tagging activity denoted as either 1 or 0 . Whereas $\hat{y}_{\text {uit }}$ is the prediction score of user $u$ for annotating item $i$ using tag $t$. Meanwhile, $w_{\text {uit }}$ is the weighted reward or penalty value assigned to $y_{\text {uit }}$.
We use CP tensor-based factorization technique to infer the latent relationship within the tensor model $\mathcal{Y}$. This technique requires less memory and time consumptions than Tucker [23]. CP factorizes tensor $\mathcal{Y} \in \mathbb{R}^{Q \times R \times S}$ into a sum of rank-one factors of $m_{f}^{(1)} \in \mathbb{R}^{Q}, m_{f}^{(2)} \in \mathbb{R}^{R}$, and $m_{f}^{(3)} \in \mathbb{R}^{S}$ for $f=$ $1, \ldots, F$. The prediction score of user $u$ to annotate item $i$ using tag $t$ is calculated as:

$$
\hat{y}_{\text {uit }}=\sum_{f=1}^{F} \prod_{n=1}^{3} m_{c_{n} f}^{(n)}=\llbracket M^{(1)}, M^{(2)}, M^{(3)} \rrbracket(9)
$$

where $c \in\{u, i, t\}$ and $F$ is the size of the latent factors. Note that for brevity, we later use $w, y$ and $\hat{y}$ to denote $w_{\text {uit }}, \hat{y}_{\text {uit }}$ and $\hat{y}_{\text {uit }}$, respectively.

\subsubsection{Generating latent factors}

Generating the latent factors $M^{(1)}, M^{(2)}$, and $M^{(3)}$, are conducted by optimizing the objective function in Eq. (8). The gradients of weighted loss function are formulated as follows:

$$
\begin{gathered}
\frac{\partial L}{\partial \theta}=\frac{\partial}{\partial \theta}\left(\sum_{u=1}^{Q} \sum_{i=1}^{R} \sum_{t=1}^{S} w \cdot \ell(y, \hat{y})\right) \\
\frac{\partial L}{\partial \theta}=\sum_{u=1}^{Q} \sum_{i=1}^{R} \sum_{t=1}^{S} w \frac{\partial \ell(y, \hat{y})}{\partial \theta}
\end{gathered}
$$

This paper uses the quadratic loss as it is the most common optimization criterion for a learning model populated from the Boolean scheme [23]. Nevertheless, the gradient of Eq. (11) can accommodate any loss function. The formulation of the quadratic loss is:

$$
\ell^{q}(y, \hat{y})=\frac{1}{2}(y-\hat{y})^{2}
$$

The quadratic loss gradient can then be formulated as:

$$
\begin{gathered}
\frac{\partial \ell^{q}(y, \hat{y})}{\partial m_{c_{n}}^{(n)}}=w \frac{\partial\left((y-\hat{y})^{2}\right)}{\partial m_{c_{n}}^{(n)}} \\
\frac{\partial \ell^{q}(y, \hat{y})}{\partial m_{c_{n}}^{(n)}}=2 \cdot w \cdot(y-\hat{y}) \frac{\partial(y-\hat{y})}{\partial m_{c_{n}}^{(n)}} \\
\frac{\partial \ell^{q}(y, \hat{y})}{\partial m_{c_{n}}^{(n)}}=-2 \cdot w \cdot(y-\hat{y}) \prod_{\substack{d=1 \\
d \neq n}}^{3} m_{c_{d} f}^{(d)}
\end{gathered}
$$

where $c \in\{u, i, t\}$ and $n \in\{1,2,3\}$. The learning algorithm of WRank is presented in Fig. 4. 


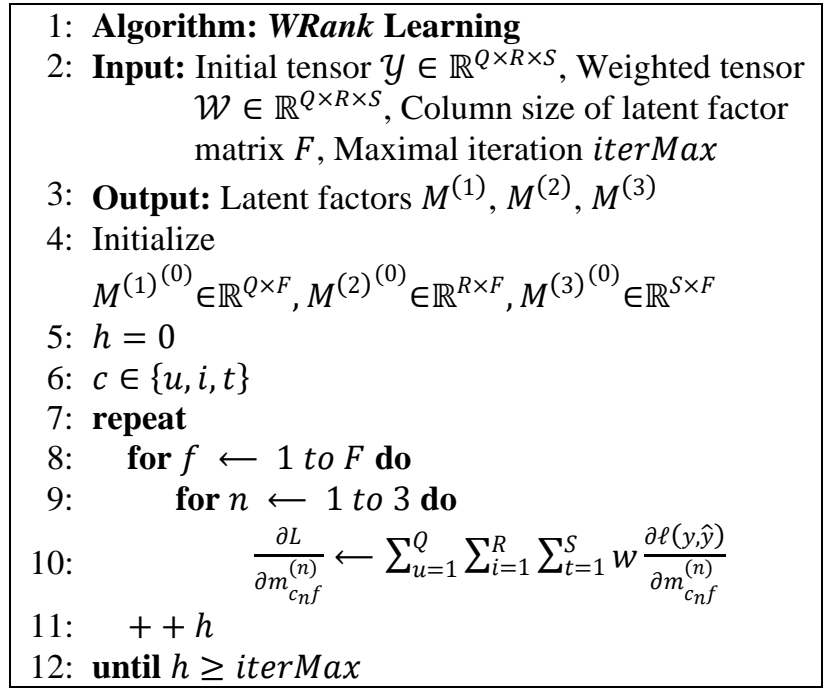

Figure. 4 The WRank learning algorithm

\subsection{Recommendation generation}

The list of item recommendations are generated based on the maximum value of $\hat{y}_{\text {uit }}$, calculated according to Eq. (9).

\section{Experiment setup}

\subsection{Datasets and evaluation method}

For the experiments, we use two real-world tagging datasets commonly used in recommendation researches, i.e., LastFM [24] and CiteULike retrieved from the CiteULike corpus (http://static.citeulike.org/data/current.bz2).

We filter the datasets using $p$-core technique [25] to remove the noise and reduce the data sparsity [6, 26]. This paper implements a variety of $p$-cores to avoid bias and maximizing the likelihood to produce stable results in the experiments [27]. The details of the datasets after the implementation of various $p$ cores implementation are shown in Table 1.

The 5-fold cross-validation technique is implemented to randomly divide each core of each dataset into an $80 \%$ training set $D_{\text {train }}$ and a $20 \%$ test set $D_{\text {test }}$ according to the number of posts. There is no overlap post between $D_{\text {train }}$ and $D_{\text {test }}$ such that no triplets of a user-item set exist in $D_{\text {train }}$ when there are triplets of $(u, i, *)$ present in $D_{\text {test }}$. $D_{\text {train }}$ is used as the learning model for generating the list of top- $N$ item predictions for the users in $D_{\text {test }}$. The recommendation performances are evaluated and reported over the average scores of all folds using AP and NDCG evaluation metrics.
Table 1. The details of datasets

\begin{tabular}{|l|c|c|c|c|c|c|}
\hline \multirow{2}{*}{$\begin{array}{c}\text { Data- } \\
\text { set }\end{array}$} & $\begin{array}{c}\boldsymbol{p} \text { - } \\
\text { core }\end{array}$ & $\begin{array}{c}\text { \#user } \\
(\boldsymbol{Q})\end{array}$ & $\begin{array}{c}\text { \#item } \\
(\boldsymbol{R})\end{array}$ & $\begin{array}{c}\text { \#tag } \\
(\boldsymbol{S})\end{array}$ & $\begin{array}{c}\text { Observed } \\
\text { Entries } \\
\left(\left|\boldsymbol{A}_{\boldsymbol{o b}}\right|\right)\end{array}$ & $\begin{array}{l}\text { Density } \\
(\boldsymbol{\%})\end{array}$ \\
\hline \hline \multirow{2}{*}{$\begin{array}{l}\text { Last- } \\
\text { FM }\end{array}$} & 10 & 867 & 1,715 & 1,423 & 99,211 & 0.0047 \\
\cline { 2 - 7 } & 15 & 703 & 1,018 & 1,063 & 76,808 & 0.0100 \\
\cline { 2 - 7 } & 20 & 601 & 681 & 838 & 61,739 & 0.0180 \\
\hline \hline \multirow{2}{*}{$\begin{array}{l}\text { Cite- } \\
\text { ULike }\end{array}$} & 10 & 1,129 & 548 & 2,403 & 17,161 & 0.0012 \\
\cline { 2 - 7 } & 15 & 721 & 203 & 1,334 & 8,099 & 0.0042 \\
\cline { 2 - 7 } & 20 & 529 & 89 & 844 & 4,254 & 0.0100 \\
\hline
\end{tabular}

Table 2. Densities comparison of $\mathcal{Y}$ and $\mathcal{W}(v=10)$

\begin{tabular}{|c|c|c|c|c|}
\hline \multirow{3}{*}{ Data-set } & \multirow{2}{*}{ Tensor } & \multicolumn{3}{|c|}{$\begin{array}{c}\text { Density of Non-zero } \\
\text { entries on } \boldsymbol{D}_{\text {train }}(\%)\end{array}$} \\
\cline { 3 - 5 } & & $\begin{array}{c}\mathbf{1 0 -} \\
\text { core }\end{array}$ & $\begin{array}{c}\mathbf{1 5}- \\
\text { core }\end{array}$ & $\begin{array}{c}\mathbf{2 0 -} \\
\text { core }\end{array}$ \\
\hline \hline \multirow{4}{*}{ Last-FM } & $\mathcal{Y}$ & 0.0042 & 0.0092 & 0.0163 \\
\cline { 2 - 5 } & $\mathcal{W}$ of WRank- $Q$ & 0.0302 & 0.0640 & 0.1122 \\
\cline { 2 - 5 } & $\mathcal{W}$ of WRank-TP & 0.0302 & 0.0640 & 0.1122 \\
\cline { 2 - 5 } & $\mathcal{W}$ of WRank-TN & 0.0183 & 0.0389 & 0.0686 \\
\hline \hline \multirow{3}{*}{ ULike- } & $\mathcal{Y}$ & 0.0010 & 0.0037 & 0.0095 \\
\cline { 2 - 5 } & $\mathcal{W}$ of WRank- $Q$ & 0.0087 & 0.0309 & 0.0808 \\
\cline { 2 - 5 } & $\mathcal{W}$ of WRank-TP & 0.0087 & 0.0309 & 0.0808 \\
\cline { 2 - 5 } & $\mathcal{W}$ of WRank-TN & 0.0049 & 0.0182 & 0.0480 \\
\hline
\end{tabular}

\subsection{Variant of the proposed WRank method}

We generate three variants of WRank to evaluate the performance of the three proposed weighting schemes. They are labelled as WRank-Q, WRank-TP, and WRank-TN to respectively following the implementation of the Quaternary-Tag-Preference, Ternary-Positive-Tag-Preference, and TernaryNegative-Tag-Preference weighting schemes to WRank. Table 2 lists the non-zero entries densities of $\mathcal{Y}$ and $\mathcal{W}$ of WRank-Q, WRank-TP and WRank-TN where $v=10$. Note that the density of WRank-Q is always equivalent to that of WRank-TP. Meanwhile, the density of WRank-TN is always less than its counterparts.

The factor matrices of the model are randomly initialized following the common approach [28]. The non-linear conjugate gradient (NCG) method of Poblano Toolbox [29] is used as the optimization method. We empirically tuned the parameters as tolerance $=1 e^{-05}$ and regularization $=0$. 


\section{Results and discussion}

\subsection{Performance comparison}

Fig. 5 and Fig. 6 respectively show the performance comparisons on the LastFM and CiteULike datasets. Recall that our proposed methods are benchmarked with the following methods: (i) HOSVD [6] where the parameters are set as tolerance $=1 e^{-04}$ and regularization $=0$; (ii) PITF [11] where the parameters are set as learning rate $=0.01$ and regularization $=$ $5 e^{-05}$; (iii) TRPR [7] where the parameters are set as tolerance $=1 e^{-04}$, regularization $=0$ and voc_size $=20$; (iv) Do-Rank [12] where the parameters are set as learning rate $=0.01$ and regularization $=1 e^{-05}$, and (v) CP-WOPT [18] where the parameters are set as tolerance $=1 e^{-05}$ and regularization $=0$. Note that we set $F=128$

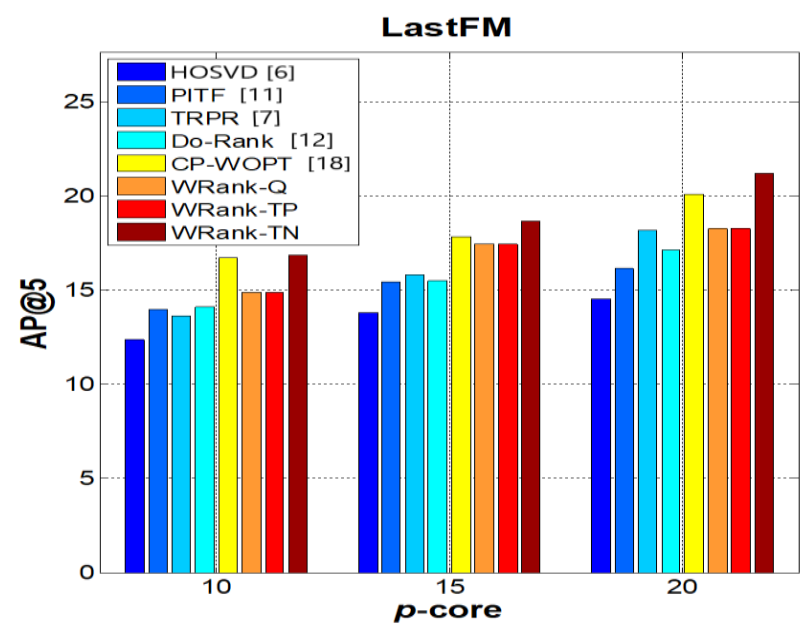

(a) for all methods, including ours. We discuss four observations based on those results.

First, the proposed WRank-TN outperforms WRank- $Q$ and WRank-TP, in terms of AP and NDCG on all datasets and $p$-cores. Interestingly, the performance comparison results contradict the density comparison results listed in Table 2, i.e., the densities of WRank-TN is the least yet it performs the best. These results indicate that the weighting schemes of WRank- $Q$ and WRank-TP include unnecessary relationships, impacting the recommendation quality as well as causing extra computation cost. On the other hand, the performance equality of WRank- $Q$ and WRank-TP, that comprehends their equal densities, indicates that the variation of entry values used to label the observed data and unobserved positive tag preference set has no impact on performances. Moreover, including the entries of unobserved positive tag preference set in

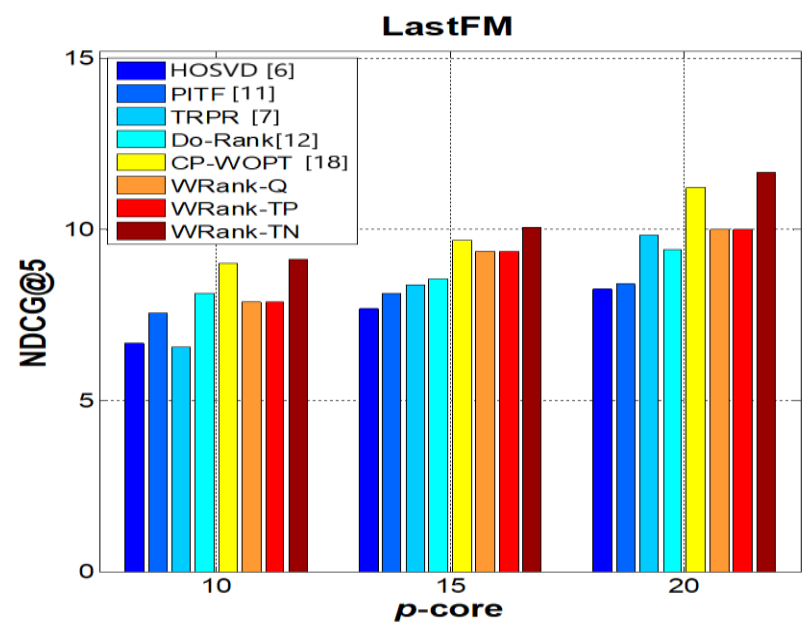

(b)

Figure. 5: (a) AP@5 and (b) NDCG@5 on LastFM dataset

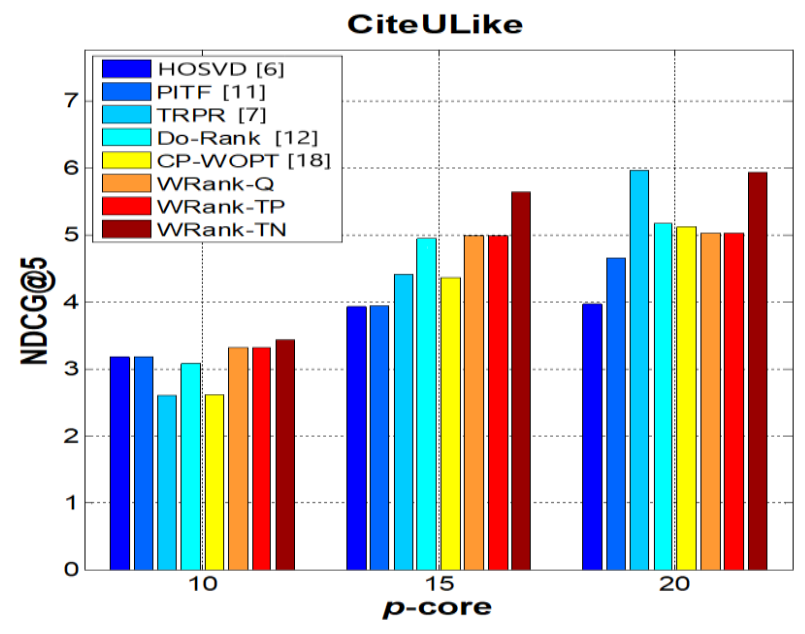

(a) AP@5

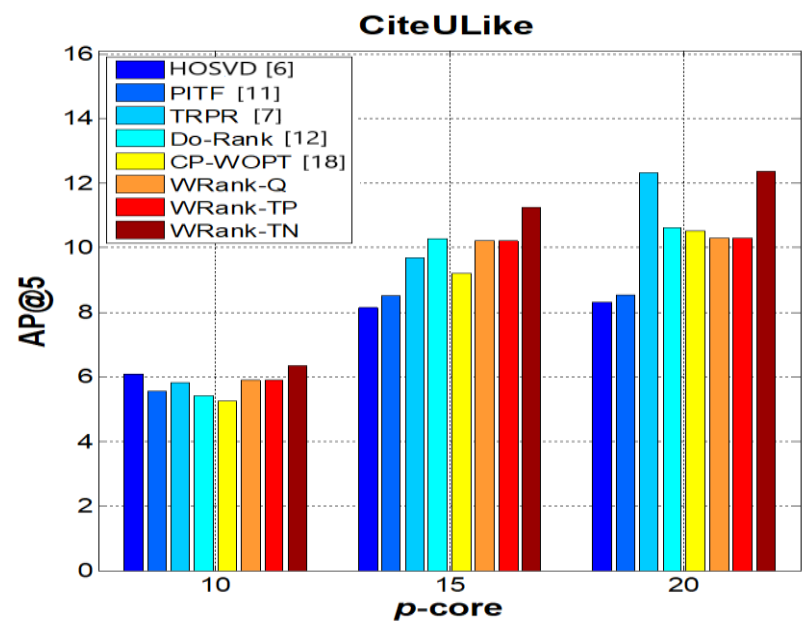

(b) NDCG@5

Figure. 6: (a) AP@5 and (b) NDCG@5 on CiteULike dataset 
the weighted-learning approach is impractical since it deteriorates the recommendation quality. In this case, we can conclude that the user tag preference is best represented as combination entries of the observed data and unobserved negative tag preference set only. Henceforward, we use WRank-TN in the discussion comparison with the benchmarking methods.

Second, WRank-TN outperforms HOSVD, TRPR, PITF, and Do-Rank. HOSVD and TRPR underperformances are because they directly learn from a model $\mathcal{Y}$ that does not exploit the data sparsity. Though TRPR implements a probabilistic ranking approach, yet the procedure is executed following, and not during, the learning process. Meanwhile, PITF and Do-Rank respectively implement the setbased and UTS schemes as a weighted-learning approach, exploiting the data sparsity, for building $\mathcal{Y}$. However, the exploitation is conducted before, instead of during, the learning process. These results ascertain that the implementation of the weightedlearning approach that presenting $\mathcal{Y}$ as binary data and uses granular $\mathcal{W}$ results in better recommendation quality compared to those that present $\mathcal{Y}$ as granular data but with no usage of $\mathcal{W}$.

Third, WRank-TN outperforms CP-WOPT in terms of both AP and NDCG on all datasets and $p$ cores. Recall that WRank-TN implements a weighting scheme that takes into account the user tag preference. In this case, WRank-TN can assign reward or penalty to both the observed and unobserved entries of $y$ during the learning process, i.e. leveraging the concept of the weighted-learning approach. In this way, the collaborative method is brined more effectively into the recommendation. On the other hand, $C P-W O P T$ uses a Boolean weighting scheme that only focuses on rewarding the observed entries of the tensor model and neglects the fact that users may have different user tag preferences. In short, this observation confirms that the users tag preferences that taking into account both the observed and unobserved entries in determining the weighting values benefits the learning process and enhances the recommendation quality.

We can also observe that WRank-TN has more dominant outperformance over $C P$-WOPT on the CiteULike dataset compared to the LastFM dataset. The reason for this can be revealed from the users' tagging behaviours of the two datasets. Recall that tag-based systems permit their users to use various tags to annotate an item as well as to use a tag to annotate numerous items. Thus, we can observe the users' tagging behaviours through the dominancy of either the observed user-item set or the user-tag set in the tagging data [30]. The user-item set is dominant

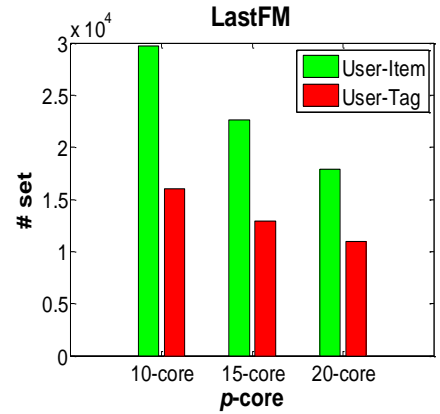

(a)

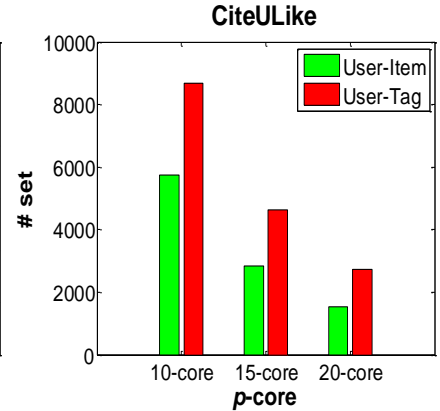

(b)
Figure. 7 The comparison of user-item and user-tag sets: (a) LastFM and (b) CiteULike

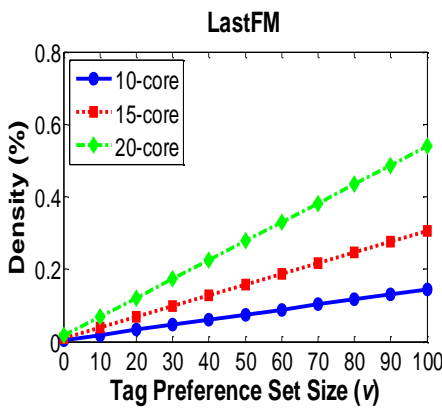

(a)

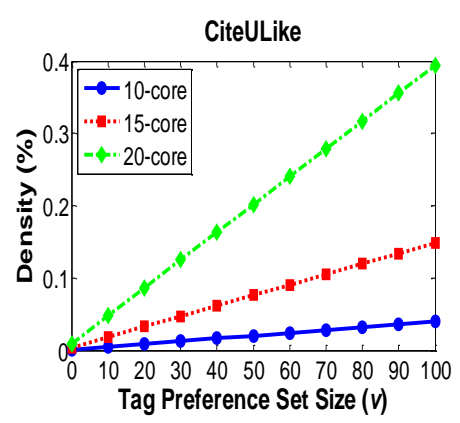

(b)
Figure. 8 The densities of tensor $\mathcal{W}$ : (a) LastFM and (b) CiteULike
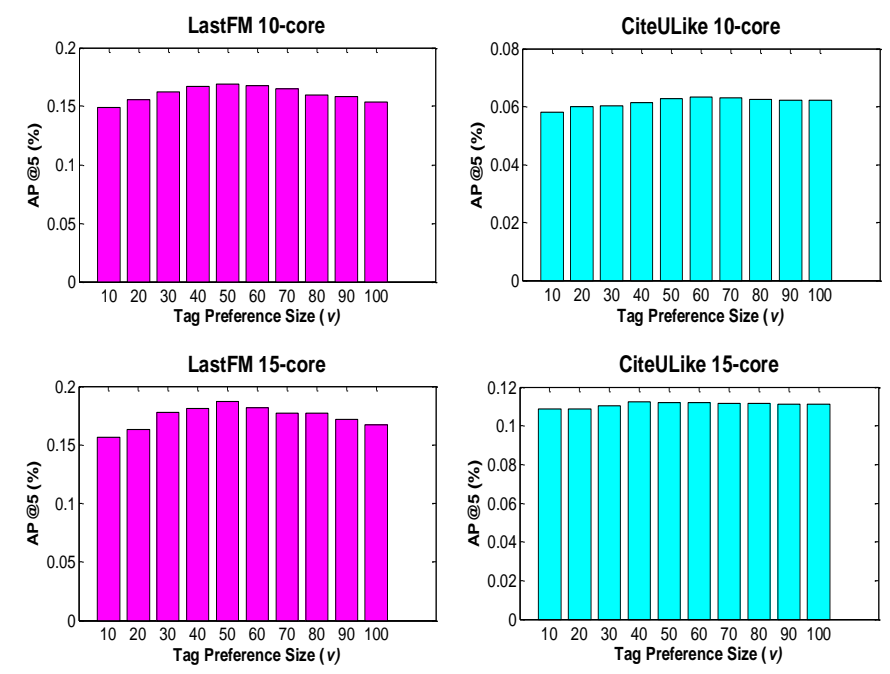

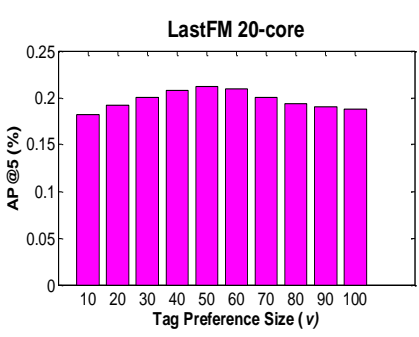

(a)

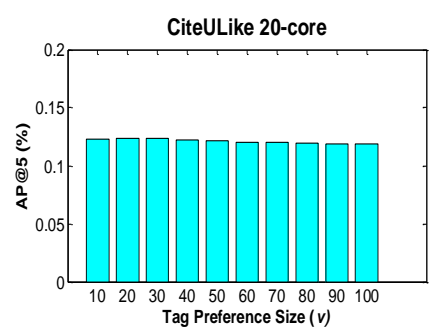

(b)
Figure. 9 The impact of the size of the user tag preference set: (a) LastFM and (b) CiteULike 
when the users are typically using very few tags when annotating items. Contrariwise, the user-tag set is dominant when users are using plenty of tags for annotating items. Fig. 7 shows that the LastFM dataset has a less dominant user-tag set than that of user-item. In contrast, the user-tag set of CiteULike dataset is more dominant than its user-item set. This points out that the weighting scheme characteristic of WRank-TN works in its favour to a dataset with a dominant user-tag set.

\subsection{Sensitivity analysis of WRank}

The sensitivities of WRank-TN are examined based on the following terms: (i) the impact of the size of $p$-core towards its quality of performance, and (ii) the impact of the size of user tag preference, $v$, towards the density of $\mathcal{W}$ and the quality of performance.

From Fig. 5 and Fig. 6, we can observe that the size of the $p$-core is linear to the performances of WRank-TN on both AP and NDCG. In other words, the bigger the $p$-core size, the higher the performance quality is. This result indicates the robustness of WRank-TN over the $p$-core refinement procedure.

Fig. 8 shows that the density of $\mathcal{W}$ is linear to $v$ on the LastFM and CiteULike datasets. We can observe that a denser $\mathcal{W}$ can always be constructed out of a sparse $\mathcal{Y}$. This observation confirms that the implementation of $\mathcal{W}$ can exploit the sparsity within $\mathcal{Y}$. Meanwhile, Fig. 9 shows that WRank-TN performs the best when $v$ is between 20 to 60 and inclines afterwards. This result suggests that the overuse of tags deteriorates the preference of users.

\section{Conclusion}

We have presented a new weighted-learning approach for exploiting data sparsity in the tag-based item recommendation system. We propose a technique to generate the user tag preference representation that leads to the formulation of three proposed weighting schemes, i.e., Quaternary-TagPreference, Ternary-Positive-Tag-Preference, and Ternary-Negative-Tag-Preference. We also proposed WRank, a weighted-learning item recommendation method to implement our proposed weighting schemes. Our experimental results show that WRank-TN, i.e., WRank that implements the Ternary-Negative-Tag-Preference weighting scheme, acquires the best performance in comparison to other proposed weighting schemes. This finding points out that the observed entries of $\mathcal{Y}$ themselves sufficiently represent the user's positive tag preference and that the user's negative tag preference is efficiently revealed from the unobserved entries of $\mathcal{Y}$. Results correspondingly show that the proposed WRank-TN method outcompetes the benchmarking methods, i.e., HOSVD, TRPR, PITF, Do-Rank, CP-WOPT. These outcomes confirm that the users' tag preferences that take into account both the observed and unobserved entries in determining the weighting values benefits the learning process and enhances the recommendation quality. Additionally, the empirical analysis also shows that WRank-TN can solve the data sparsity problem and is robust over $p$-core refinement procedure.

\section{Conflicts of Interest}

We declare that there is no conflict of interest in this work.

\section{Author Contributions}

The details of authors contributions are: "Conceptualization, Noor Ifada; methodology, Noor Ifada; software, Noor Ifada; validation, Noor Ifada and Richi Nayak; formal analysis, Noor Ifada and Richi Nayak; investigation, Noor Ifada; resources, Noor Ifada; data curation, Noor Ifada; writingoriginal draft preparation, Noor Ifada; writingreview and editing, Noor Ifada and Richi Nayak; visualization, Noor Ifada; supervision, Noor Ifada and Richi Nayak; project administration, Noor Ifada; funding acquisition, Noor Ifada and Richi Nayak".

\section{References}

[1] T. Bogers, "Tag-based recommendation", Social Information Access, Springer, Cham, pp. 441479, 2018.

[2] C. C. Aggarwal, Recommender Systems: The Textbook, Springer International Publishing, Switzerland, 2016.

[3] E. El Sayed, A. El Korany, A. Salah, and H. Hefny, "Exploiting Social Annotations for Personalizing Retrieval", International Journal of Intelligent Engineering and Systems, Vol. 10, No. 6, pp. 192-202, 2017.

[4] E. E. S. Mahmoud and A. S. Mohamed, "An Ontology Based Framework for Automatic Web Resources Identification", International Journal of Intelligent Engineering and Systems, Vol. 13, No. 2, pp. 299-306, 2020.

[5] E. Frolov and I. Oseledets, "Tensor methods and recommender systems", Wiley Interdisciplinary Reviews: Data Mining and Knowledge Discovery, Vol. 7, No. 3, p. e1201, 2017.

[6] P. Symeonidis, A. Nanopoulos, and Y. Manolopoulos, "A Unified Framework for 
Providing Recommendations in Social Tagging Systems Based on Ternary Semantic Analysis", IEEE Transactions on Knowledge and Data Engineering, Vol. 22, No. 2, pp. 179-192, 2010.

[7] N. Ifada and R. Nayak, "A Two-Stage Item Recommendation Method Using Probabilistic Ranking with Reconstructed Tensor Model", in User Modeling, Adaptation, and Personalization, Springer, Cham, pp. 98-110, 2014.

[8] A. Klašnja-Milićević, M. Ivanović, B. Vesin, and Z. Budimac, "Enhancing e-learning systems with personalized recommendation based on collaborative tagging techniques", Applied Intelligence, Vol. 48, No. 6, pp. 1519-1535, 2018.

[9] Y. Yang, L. Han, Z. Gou, B. Duan, J. Zhu, and H. Yan, "Tagrec-CMTF: Coupled matrix and tensor factorization for tag recommendation", IEEE Access, Vol. 6, pp. 64142-64152, 2018.

[10] X. Tang, Y. Xu, and S. Geva, "Factorizationbased primary dimension modelling for multidimensional data in recommender systems", International Journal of Machine Learning and Cybernetics, Vol. 10, No. 8, pp. 2209-2228, 2019.

[11] S. Rendle and L. Schmidt-Thieme, "Pairwise Interaction Tensor Factorization for Personalized Tag Recommendation", In: Proc. of The 3rd ACM International Conf. on Web Search and Data Mining, New York, USA, pp. 81-90, 2010.

[12] N. Ifada and R. Nayak, "Do-Rank: DCG Optimization for Learning-to-Rank in TagBased Item Recommendation Systems", Advances in Knowledge Discovery and Data Mining, Springer International Publishing, pp. 510-521, 2015.

[13] H. Lim and H. J. Kim, "Item recommendation using tag emotion in social cataloging services", Expert Systems with Applications, Vol. 89, pp. 179-187, 2017.

[14] H. Lim and H. J. Kim, "Tensor-based tag emotion aware recommendation with probabilistic ranking", KSII Transactions on Internet and Information Systems (TIIS), Vol. 13, No. 12, pp. 5826-5841, 2019.

[15] H. Zhang, Q. Hong, X. Shi, and J. He, "A Social Tagging Recommendation Model Based on Improved Artificial Fish Swarm Algorithm and Tensor Decomposition", Advances in Intelligent Systems and Computing, Vol. 733, Springer, Cham, pp. 3-13, 2018.

[16] K. Hosono, S. Ono, and T. Miyata, "Weighted tensor nuclear norm minimization for color image denoising", In: Proc. of International Conf. on Image Processing (ICIP), Phoenix, AZ, USA, pp. 3081-3085, 2016.

[17] Y. Mu, P. Wang, L. Lu, X. Zhang, and L. Qi, "Weighted tensor nuclear norm minimization for tensor completion using tensor-SVD", Pattern Recognition Letters, Vol. 130, pp. 4-11, 2020.

[18] E. Acar, D. M. Dunlavy, T. G. Kolda, and M. Mørup, "Scalable Tensor Factorizations for Incomplete Data", Chemometrics and Intelligent Laboratory Systems, Vol. 106, No. 1, pp. 41-56, 2011.

[19] M. Filipović and A. Jukić, "Tucker factorization with missing data with application to low-n-rank tensor completion", Multidimensional Systems and Signal Processing, Vol. 26, No. 3, pp. 677692, 2015.

[20] L. Yuan, Q. Zhao, and J. Cao, "Completion of high order tensor data with missing entries via tensor-train decomposition", In: Proc. of International Conf. on Neural Information Processing, Guangzhou, China, pp. 222-229, 2017.

[21] M. Iwata, L. Yuan, Q. Zhao, Y. Tabei, F. Berenger, R. Sawada, S. Akiyoshi, et al., "Predicting drug-induced transcriptome responses of a wide range of human cell lines by a novel tensor-train decomposition algorithm", Bioinformatics, Vol. 35, No. 14, pp. i191-i199, 2019.

[22] J. Kim, Y. He, and H. Park, "Algorithms for Nonnegative Matrix and Tensor Factorizations: A Unified View based on Block Coordinate Descent Framework", Journal of Global Optimization, Vol. 58, No. 2, pp. 285-319, 2014.

[23] T. Kolda and B. Bader, "Tensor Decompositions and Applications", SIAM Review, Vol. 51, No. 3, pp. 455-500, 2009.

[24] I. Cantador, P. Brusilovsky, and T. Kuflik, "Second Workshop on Information Heterogeneity and Fusion in Recommender Systems (HetRec2011)", In: Proc. of the 5th ACM Conf. on Recommender Systems, Chicago, Illinois, USA, pp. 387-388, 2011.

[25] V. Batagelj and M. Zaveršnik, "Generalized Cores", arXiv Preprint cs/0202039, 2002.

[26] D. Rafailidis and P. Daras, "The TFC Model: Tensor Factorization and Tag Clustering for Item Recommendation in Social Tagging Systems", IEEE Transactions on Systems, Man and Cybernetics, Part A: Systems and Humans, Vol. 43, No. 3, pp. 673-688, 2013.

[27] S. Doerfel and R. Jäschke, "An Analysis of Tagrecommender Evaluation Procedures”, In: Proc. 
of The 7th ACM Conf. on Recommender Systems, pp. 343-346, 2013.

[28] S. Balakrishnan and S. Chopra, "Collaborative Ranking", In: Proc. of the 5th ACM International Conf. on Web Search and Data Mining, Seattle, Washington, USA, pp. 143-152, 2012.

[29] D. M. Dunlavy, T. G. Kolda, and E. Acar, Poblano v1.0: A Matlab Toolbox for GradientBased Optimization, Sandia National Laboratories, Albuquerque, NM and Livermore, CA, SAND2010-1422, 2010.

[30] N. Ifada and R. Nayak, "An Efficient Tagging Data Interpretation and Representation Scheme for Item Recommendation", In: Proc. of the 12th Australasian Data Mining Conf., Brisbane, Australia, pp. 205-215, 2014. 